\title{
Erratum
}

\section{Compact Differentiable 4-Folds with Quaternionic Structures}

Ma. Kato

Department of Mathematics, Sophia University, Kioi-cho, Chiyoda-ku, Tokyo 102, Japan

Math. Ann. 248, 79-96 (1980)

The statement of Proposition 8 in "compact differentiable 4-folds with quaternionic structures", was incomplete and a subcase was missing. We add the following subcase to Proposition 8.

(v) $\quad\left\{B_{2}, c v_{B_{2}}\right\}=\left(K \times\left\{c^{3} I\right\}\right) \cup\left(c v_{B_{2}}\right)\left(K \times\left\{c^{3} I\right\}\right) \cup\left(c v_{B_{2}}\right)^{2}\left(K \times\left\{c^{3} I\right\}\right)$, where $c \in \mathbf{R}, 0<c<1$, and $v_{B_{2}}=\frac{1}{\sqrt{2}}\left(\begin{array}{cc}\varrho_{4}^{3} & \varrho_{4}^{3} \\ \varrho_{4} & -\varrho_{4}\end{array}\right)$.

Therefore, p. $95 \uparrow 8$, "... are involutions of $S^{3} / H$ " should read as "...are of order 2 or 3 as an element of the diffeomorphism group of $S^{3} / H^{\prime \prime}$. The error was in (41). In case $K=B_{2}$, the possibility of $(\operatorname{det} g)^{-1 / 2} g=v_{B_{2}}$ was missing. This error had its origin in that of Lemma 4 in my previous paper "Topology of Hopf surfaces", J. Math. Soc. Japan 27, 222-238 (1975). The correction of that paper will appear in J. Math. Soc. Japan.

Acknowledgement. I am very much grateful to M. Ue who kindly informed me about the mistake.

Received October 17, 1988 